\title{
Cuidando las 24 horas del día. Un estudio de caso sobre cuidadoras familiares y no familiares en la comarca de La Garrotxa (Girona)
}

\author{
Xavier Casademont ${ }^{1}$
}

Recibido: 26/06/2017/ Revisado: 27/06/2017 / Aceptado: 23/11/2017

Disponible on line

Resumen. La situación de las cuidadoras familiares y no familiares que dedican las 24 horas del día al cuidado de las personas dependientes es una realidad a menudo oculta en la sociedad. Este artículo analiza la situación de las cuidadoras mediante un estudio de caso realizado en la comarca de La Garrotxa (Girona), una de las comarcas con índices de envejecimiento y sobre-envejecimiento más elevados de Cataluña, así como con un elevado porcentaje de población extranjera, que en algunos casos, se han dedicado a los cuidados de forma remunerada. La metodología utilizada es principalmente cualitativa. Se han realizado 20 entrevistas en profundidad, 10 a cuidadoras familiares y 10 a cuidadoras no familiares, así como un grupo de contraste con trabajadoras sociales. Los resultados muestran que las personas que se dedican las 24 horas del día al cuidado de las personas dependientes sufren unas condiciones de vida duras que las limitan especialmente en su vida personal y comunitaria, y que afectan de manera notable a su estado de salud. La mayoría de ellas lleva períodos prolongados de tiempo asumiendo las cargas de cuidado, con lo cual su situación es especialmente vulnerable. Entre las cuidadoras familiares destaca una sensación generalizada de agotamiento por desempeñar una tarea escasamente reconocida y para la cual no cuentan con demasiadas ayudas. Entre las cuidadoras no familiares son especialmente duras las condiciones de trabajo, estimuladas por la situación administrativa irregular, que no les deja margen para disponer de mejores condiciones o para trabajar en otros ámbitos. Palabras clave: cuidados, dependencia, desigualdad, inmigración, mujeres.

\section{[en] 24-hour care: a case study with family and non-family caregivers in the region of La Garrotxa (Girona)}

Abstract. The situation of family and non-family caregivers who provide 24-hour daily care to dependants is an oftenhidden reality in society. This article analyses the circumstances of caregivers by means of a case study conducted in the region of La Garrotxa (Girona), one of the regions of Cataluña with the highest levels of old age and superannuation, as well as a high immigrant population that has dedicated itself in some cases to remunerated caregiving. The methodology used was principally qualitative, with 20 in-depth interviews conducted - ten with family caregivers and ten with nonfamily caregivers, as well as a comparison group of social workers. The results show that people who dedicate 24 hours per day to caring for dependant persons experience difficulty living conditions that place particular limits on their personal and social lives, and that have a notable impact on their state of health. The majority of these caregivers have been engaged in their roles for lengthy periods of time, making their situation especially vulnerable. Family caregivers emphasise a general sense of exhaustion due to performing a task with little recognition and a limited amount of support. Working conditions are particularly tough for non-family caregivers, stimulated by their irregular administrative circumstances that leave no room for access to improved conditions or work in different areas.

Key words: care, dependency, inequality, immigration, women

Sumario: Introducción. 1. Metodología. 2. Las cuidadoras no familiares. 3. Las cuidadoras no familiares. 4. Conclusiones. 5. Referencias bibliográficas.

Cómo citar: Casademont, X. (2019). Cuidando las 24 horas del día. Un estudio de caso sobre cuidadoras familiares y no familiares en la comarca de La Garrotxa (Girona), en Cuad. trab. soc. 32(1), 61-70.

\footnotetext{
Universitat de Girona, España

Consorci de Acció Social de La Garrotxa, España

xavier.casademont@udg.edu
} 


\section{Introducción}

Una de las características de la condición humana es la vulnerabilidad. El apoyo y las tareas de cuidado son necesarias a lo largo de diferentes etapas y situaciones vitales: la infancia, el envejecimiento y la enfermedad son tres de los casos más habituales. Y es que, en el devenir de la vida, los seres humanos necesitan que otras personas cuiden de ellas y, por lo tanto, la condición de dependientes no se puede considerar una excepcionalidad, sino un elemento intrínseco de la especie humana (Rogero, 2009ª; Tobío, Agulló Tomás, Gómez y Martín Palomo, 2010). Ante esta realidad, son varios los actores que pueden intervenir y conformar un determinado régimen de bienestar: el Estado, el mercado, la familia y otras instituciones sociales, especialmente las vinculadas al tercer sector social (Esping-Andersen, 1999; Rodríguez Cabrero, 2011; Torns, Borrás, Moreno y Recio, 2012). En el caso español la presencia femenina es mayoritaria y genera fuertes desigualdades (Lagarde, 2004; Abellán García, Esparza Catalá y Pérez Díaz, 2011). En todas las etapas vitales las personas experimentan su condición de fragilidad y dependencia, así como la posibilidad de ser sujetos activos o pasivos de los cuidados (Rogero, 2009b). Sin embargo, el redescubrimiento del trabajo de cuidados (Carrasquer, 2013) plantea interrogantes sobre la visibilidad del trabajo femenino, su contribución en la provisión de bienestar, la división sexual del trabajo y las desigualdades de género (Larrañaga Martín, Bacigalupe, Begiristáina, Valderrama, y Arregi, 2008), etc., especialmente en los regímenes de bienestar mediterráneos (Ferrera, 1996; Navarro, 2002).

La extensión del sistema catalán de autonomía y atención a la dependencia, a partir de la aprobación de la Ley 39/2006, de Promoción de la Autonomía Personal y Atención a las personas en situación de dependencia, y de la Ley 12/2007, de servicios sociales de Cataluña, favorecía que las situaciones de falta de autonomía se atendieran en el domicilio, y que las personas que se ocuparan de ello recibieran una prestación económica (Vilà, 2013; Aguilar Hendrickson, 2014). La prestación económica para cuidadores suponía un paso importante hacia el reconocimiento de las tareas de cuidado (Generalitat de Catalunya, 2008) y, al mismo tiempo, favorecía la permanencia en el domicilio en detrimento del ingreso en centros residenciales, una opción preferida para la mayoría de la población (IMSERSO, 2005).

Son diversos los trabajos que han analizado la situación de cuidadores de personas dependientes, tanto desde una perspectiva integral (Carretero, Garcés y Rodenas, 2006; Rogero, 2009a), como desde una perspectiva específica, como los impactos en la salud (Larrañaga et al., 2008), del trabajo (Torns, 2008), en términos de desigualdad de género (Campo Ladero, 2000), en colectivo sociales (Colectivo IOE, 2005) o en relación con el Trabajo Social (Fuentes y Moro, 2014). La mayoría de ellos se basa en aproximaciones teóricas o en análisis estadísticos del conjunto del Estado o de regiones españolas. Por el contrario, esta investigación de tipo micro analiza un entorno local y se centra en el análisis cualitativo de las personas cuidadoras, para ofrecer un relato personal y subjetivo que, a pesar de ello, complementa aportaciones más generalistas.

El estudio de la situación de las personas cuidadoras "24 horas" analiza el caso de La Garrotxa (Girona), con 21 municipios y 56.000 habitantes, una de las comarcas catalanas con índices de envejecimiento más elevados. Para ello se estudian dos grandes perfiles de población: por un lado, las personas que se encargan de cuidar de forma no profesionalizada a un familiar en situación de dependencia, generalmente, cónyuges. Por el otro, las personas que, de forma remunerada y sin que existan vínculos familiares, cuidan a personas con limitaciones en su autonomía personal.

Los principales objetivos de esta investigación son: 1) Identificar las principales tareas que desempeñan las cuidadoras, y en qué condiciones las desarrollan; 2) Profundizar en los impactos personales y familiares de esta actividad, especialmente en términos de salud y en la dimensión relacional; y 3 ) indagar en cómo perciben que su actividad es reconocida, tanto por parte de la persona a quien cuidan, como por el resto de los familiares y de la sociedad.

\section{Metodología}

De acuerdo con los objetivos de investigación, la metodología utilizada es cualitativa y las técnicas son las entrevistas en profundidad a cuidadoras y a un grupo de contraste con profesionales de los servicios sociales expertos en atención a la dependencia. Por esta razón, porque era necesario conocer de primera mano 
las impresiones de las personas cuidadoras, se ha optado por una investigación cualitativa que nos permite captar las impresiones y las opiniones de los informantes, así como los detalles relevantes relacionados con las percepciones y el relato de las personas que, en este caso, se ocupan de las personas dependientes.

La Garrotxa combina dos casuísticas especialmente relevantes para el análisis del fenómeno de las cuidadoras: por un lado, la población mayor de 65 años supera casi el 2 por ciento la media catalana, y el porcentaje de población mayor de 85 años lo supera en 1.1 puntos. La esperanza de vida de la población de La Garrotxa es alta; y es entre las mujeres donde esta proporción es más destacada, hasta el punto de que por cada hombre mayor de 85 años hay 2.1 mujeres (Institut d'Estadística de Catalunya).

Por otro lado, La Garrotxa es una de las comarcas con mayor proporción de personas extranjeras que, en veinte años, ha aumentado del 4 al 15 por ciento de la población. La inmigración es especialmente relevante en una doble dirección: por un lado, porque puede convertirse - si no aumentan las cifras del crecimiento natural de la población - en una de las principales soluciones a los déficits que presenta la pirámide demográfica actual, especialmente reducida en las franjas de edad correspondientes a la población en edad joven y activa. Asimismo, la inmigración ha sido uno de los colectivos que ha cubierto la creciente demanda de puestos de trabajo de la estructura productiva de la comarca; pero, sobre todo, en actividades profesionales remuneradas de tipo formal e informal, vinculadas al cuidado y la atención a las personas en situación de dependencia (Bettio, Simonazzi y Villa, 2006; Anderson, 2014).

En total se ha entrevistado a 20 cuidadoras, 10 familiares y 10 no familiares, en base a los datos del perfil de las personas cuidadoras en España (IMSERSO, 2005). De las familiares, la mayoría eran mujeres, entre 70 y 88 años, y cuidaban de sus cónyuges. Las cuidadoras no familiares eran mujeres, entre 22 y 54 años y de nacionalidad extranjera.

A pesar de los contactos previos de los profesionales de referencia, ha sido difícil concertar las entrevistas con las cuidadoras, dada la poca flexibilidad horaria de que disponen. Este hecho, común entre las cuidadoras familiares y las no familiares, se agravaba en el caso de las no familiares, ya que suelen tener más difi- cultades para participar en actividades de tipo personal. En este caso, hay que dejar constancia de los recelos y miedos que algunas personas manifestaron a la hora de poder expresarse libremente, sobre todo porque temían que esto les pudiese perjudicar.

Se diseñaron dos cuestionarios dirigidos a los dos perfiles a entrevistar: un cuestionario para las cuidadoras familiares y otro para las cuidadoras no familiares. Los dos modelos de entrevista tuvieron en cuenta algunas de las principales consideraciones presentes en la literatura científica, así como también algunas variables que forman parte de la escala de sobrecarga del cuidador (Test de Zarit). Las principales dimensiones de análisis fueron el perfil de las cuidadoras; el perfil de las personas dependientes; las tareas, funciones y condiciones asociadas al cuidado; los impactos en la vida personal y familiar, entre otros.

Una vez se diseñó un primer borrador de los dos modelos de entrevista, se desarrolló el primer grupo de contraste, con la participación de seis profesionales: tres trabajadoras sociales del Servicio Básico de Atención Social, y 3 profesionales del equipo del Servicio de Valoración de la Dependencia de La Garrotxa, que aportaron propuestas y modificaciones. El grupo se volvió a reunir para presentar los resultados y contrastarlos con las profesionales.

\section{Las cuidadoras familiares}

Las cuidadoras familiares desarrollan un número considerable de tareas y funciones que requieren una dedicación importante. Así, las tareas para las que se necesita ayuda son: 1) tareas domésticas (limpiar el domicilio, lavar y planchar la ropa, cocinar, administrar los ingresos del hogar, etc.); 2) actividades cotidianas (levantar y acostar, salir a la calle, comprar, acompañar a visitas médicas, moverse por el domicilio, administrar la medicación, responsabilizarse de gestiones diversas, etc.); y 3) cuidados personales (ducharse, vestirse, ayudar a comer, apoyo en caso de incontinencia urinaria $y / o$ fecal, etc.).

Mi vida es levantarme, arreglarlo, hacer mis faenas de mi casa, mi comida, ir a comprar, a ratos me lo dejo acostado, si no hace mucho calor me lo llevo conmigo, y a la tarde pues estar los dos aquí, cada vez que tiene que hacer pipí pues le pongo aquí para hacer pipí, si quiere agua le 
tengo que poner agua, porque él no puede coger ni un vaso de agua, o moverlo de postura, y a la noche, igual. Nos vamos a dormir y si tengo que levantarme, tres o cuatro veces, porque él necesita que lo muevan, hacer pipí... Y ésta es mi vida (CFAM7).

Cada una de estas tareas conlleva dedicaciones e implicaciones diferentes para la cuidadora. Obviamente, no todas se asumen de manera automática, sino que progresivamente se puede ir ampliando el abanico de funciones. Hay una serie de tareas que, tradicionalmente, ya habían asumido en exclusividad las mujeres, incluso en los períodos en que la persona con la que convivían era autónoma: nos referimos a las tareas domésticas y a una parte de las actividades cotidianas. Las tareas que generan inicialmente más reticencias son las que evidencian la pérdida de autonomía de la persona dependiente, que implican más esfuerzo físico para la cuidadora. La mayoría de las cuidadoras manifiestan que el progresivo deterioro de la persona dependiente les afecta negativamente. Con el paso del tiempo, sin embargo, la práctica continuada provoca la asunción y la "familiarización" con estas actividades, que se traduce en la adquisición de automatismos que se incorporan casi de forma inconsciente en el día a día de las cuidadoras. No, no, nada, como se trata de una cosa que llevo tanto tiempo haciendo, es como una rutina, claro, hace muchos años [...] ya con mi padre lo hice, hace tantos años que ya no me molesta (CFAM2).

La sensación de sobrecarga que expresan las cuidadoras se sostiene por el vínculo familiar y afectivo con la persona a quien se cuida, y por la convicción de la responsabilidad que les corresponde en tanto que familiares y seres queridos.

Es una carga, para mi es una carga, que la hago porque es mi madre, lo tengo que hacer, yo las cosas las hago cuando las personas están vivas, no para ir al cementerio, pero para mí es una carga. O sea, cuando estoy allí no puedo salir nunca, no salgo, yo no salgo, y ya hace tiempo que estoy así, pero bueno, ahora estamos en esta situación (CFAM4).

Otra de las características relacionadas con las tareas de cuidado es la intensidad y la prolongación de la ayuda. La mayoría de las entrevistadas hace mucho tiempo que ejercen esta función con una dedicación intensa y ex- clusiva ( 3 personas desde hace más de 20 años; 4 entre 10 y 20 años y 3 menos de 10 años). Lógicamente, con el paso de los años aumentan las dificultades, y las personas con más años de dedicación expresan más cansancio, a pesar de que también son decisivas otras variables, como el estado de la persona dependiente. En esta tesitura, pues, como consecuencia del desgaste provocado por los largos períodos de cuidado, algunas cuidadoras se plantean la posibilidad de la muerte, tanto de la cuidadora como de la dependiente, lo que debe interpretarse como un síntoma de agotamiento físico y emocional.

Es un golpe muy duro que te digan que con sesenta años se va a quedar así. Y, además, que luego viva tantos años, y tú piensas, sí, 91, gracias por la compañía, pero, me entiendes, ¿no? No le quiero desear la muerte, ¿me entiendes lo que quiero decir? Pero no te esperas que un hombre así viva 92 años, y lo que puede llegar a vivir, porque tiene una salud (CFAM6)

Las actividades y tareas de las cuidadoras son cada vez más complejas y exigen una serie de habilidades, conocimientos y competencias, si se quieren desarrollar correctamente. No obstante, la mayoría de las cuidadoras señalan que la experiencia es fundamental en su formación. Ha sido el contacto directo con la realidad, las necesidades surgidas a lo largo del vínculo diario con las personas dependientes, lo que les ha servido para enfrentarse a las tareas de cuidado. En cierto modo, pues, la mayoría de las cuidadoras se consideran autodidactas y, como mucho, destacan haber aprendido ciertas habilidades mediante la relación con otros profesionales, como médicos, enfermeras, cuidadores no familiares o del ámbito de la gerontología. "Esto es como cuando somos madres, que nadie nos ha enseñado. $Y$ vas haciendo, vas preguntando, te informando, te entero de cosas, de comodidades, te vas poniendo al día" (CFAM4).

Finalmente, también vale la pena reflexionar sobre el proceso por el cual estas personas se convirtieron en las cuidadoras. Es decir: si existieron procesos de diálogo y negociación con la persona dependiente $\mathrm{y} / \mathrm{o}$ el resto de familiares, y cómo se decidió cuál sería la opción escogida. En términos generales, la opción más habitual es la asunción normalizada de que es a la mujer a quien le corresponde dedicarse a las tareas de cuidado (Lagarde, 
2004). Así lo expresan las mismas mujeres que, en algunos casos, apelaron al compromiso matrimonial. "Cuando me casé me dijeron: para lo bueno y para lo malo, y me ha tocado esto, pues tengo que hacer esto. No hay otra solución". (CAFM1). Además, las cuidadoras manifiestan una cierta voluntad de cuidar hasta que no puedan más, lo que implica, en muchos casos, un compromiso absoluto que las afecta negativamente, a medio y a largo plazo. En estas circunstancias, algunas mujeres expresan que sus creencias religiosas les resultan especialmente útiles para continuar con las tareas de cuidado y atención, ya que consideran que están cumpliendo el deber de acuerdo con sus convicciones.

Mayoritariamente, pues, se expresa una clara voluntad de quedarse a vivir en el hogar, lo que, en parte, condiciona que el cuidado corresponda a la persona con la que convive la persona dependiente. El hogar se considera el entorno más idóneo para garantizar la calidad de vida de la persona dependiente, $\mathrm{y}$, además, se verbaliza una desconfianza y una crítica severa a las supuestas condiciones de las residencias, que se tachan de impersonales y frías, donde las condiciones de vida de los residentes empeoran respecto a las de los domicilios particulares.

Por lo que respeta al reconocimiento, la mayoría manifiestan una falta de reconocimiento social, desde la perspectiva económica (porque no es un trabajo remunerado o, en caso de recibir la prestación como cuidadora familiar, se considera absolutamente insuficiente); la institucional (por la falta de medidas efectivas dirigidas a mejorar la atención de las personas dependientes); y la comunitaria (por las tímidas muestras de reconocimiento y apoyo hacia su tarea). En algunos casos se ponen como ejemplo de la insensibilidad institucional las dificultades burocráticas y administrativas vinculadas a determinados procesos administrativos que implican excesivas gestiones presenciales que alteran y distorsionan la dinámica habitual de las personas cuidadoras.

No es una cosa que te lo reconozcan y vas a los sitios a pedir una cosa y parece que vas a pedir limosna, y no se va a pedir limosna, se va a solicitar una cosa que nosotros hemos pagado trabajando [...] Yo a veces de impotencia, en los sitios, es que me entraban ganas de llorar, y los viajes que yo daba, y yo tenía que ir, pedirme a mis compañeras, porque en los trabajos yo tampoco podía faltar (CFAM7).

En cuanto al reconocimiento por parte de la persona cuidadora, a pesar de destacar un reconocimiento favorable, se formulan muchos matices que relativizan esta afirmación. En primer lugar, porque en algunos casos las personas dependientes no gozan de las condiciones físicas ni psíquicas para expresarse de manera normalizada, lo que complica especialmente las tareas de cuidado y hace imposible la emisión de opiniones y emociones fehacientes. Son muchos los casos en que se expresan episodios, más o menos esporádicos, de malos tratos, gritos y otras formas, más o menos explícitas, de violencia que, lógicamente, complican mucho más la tarea y afectan la autoestima y al ánimo de la cuidadora.

No, no, es él quien me lo tendría que reconocer, es a él a quien cuido, no a otros, y él no me lo reconoce. Porque a veces no me tendría que decir según qué cosas. A veces le digo: Soy tu criada, soy tu enfermera, lo soy todo. Pero él... manda, siempre manda.[...] No me tendría que chillar, a veces, como me chilla, porque a veces me ha levantado la mano, y me tengo que apartar, porque si no me tocaría (CFAM1).

Otra dimensión de análisis son los impactos en las cuidadoras: uno de los principales es en el ámbito laboral. Buena parte de las cuidadoras habían combinado, durante una parte importante de su vida, el trabajo productivo con el reproductivo, y la gran mayoría de ellas tuvo renunciar al trabajo productivo para poder cuidar alguna persona dependiente, ya fuera su marido o algún otro familiar. Además del truncamiento de las legítimas aspiraciones personales, tuvieron que hacer frente a la supresión de su fuente de ingresos, garantía de una cierta autonomía personal, con las implicaciones que ello supone de cara a la cuantía de la pensión de jubilación.

Sin embargo, los principales impactos se dan en las condiciones de salud. Las características intrínsecas de las tareas de cuidado tienen notables consecuencias en las condiciones físicas y psíquicas de personas que, por razones de edad sobre todo, ya tienen ciertas dificultades de salud. En bastantes casos las cuidadoras asisten a sesiones de recuperación, especialmente de fisioterapia. Así, son muy habituales diversas molestias de tipo muscular, 
especialmente en las piernas, los brazos y las articulaciones, así como también problemas de huesos (artritis y artrosis), y sobre todo en zonas concretas, como la espalda, las lumbares, columna, vértebras, etc.

Algunas incluso renuncian a intervenciones médicas que mejorarían su calidad de vida, para evitar ausentarse de las tareas de cuidado. Estos casos muestran actitudes de sacrificio personal muy elevado en el que se pone por delante el cuidado de la persona dependiente en detrimento de la propia salud. Este es el caso de una cuidadora que no quiere dejar de cuidar a su marido, ya que la operación a que se debería someter implica un período relativamente largo de recuperación. Otras, en casos menos graves, hacen todo lo que pueden para evitar estar indispuestas y poder continuar ejerciendo sus actividades de cuidado.

Además, las cuidadoras tienen problemáticas diversas que afectan de manera importante su estado anímico: períodos de angustia, estrés, tristeza, desazón, agotamiento, apatía, depresión... "Yo cogí como una angustia... es que no podía, yo me tomo la pastilla, pero es que era 1lorar, temblar y angustiada" (CFAM8). En algunos casos, el deterioro de la salud mental de las cuidadoras implica la prescripción de medicamentos.

Son varios los motivos que explican las afectaciones en el estado anímico. En primer lugar, la condición de familiar, que se intensifica en el caso de los matrimonios, y la tristeza que provoca presenciar el progresivo deterioro de las condiciones de salud de un ser querido. Asimismo, está muy presente la insatisfacción que provoca el hecho de no haber podido disfrutar activamente de una etapa importante de la vida en que, en caso de gozar de buena salud, las personas mayores pueden vivir con plenitud y realizar actividades de ocio.

Todo ello también afecta de manera destacada a su vida social y la participación en otras actividades. La condición de cuidadora implica un proceso de aislamiento social, de desaparición de determinados espacios de socialización y ocio, más allá del ámbito doméstico. "Sé que tengo muy poca vida, la vida que tengo la tengo dentro de mi casa [...] estoy las 24 horas del día pendiente de él" (CFAM8).

Este hecho lo viven especialmente de forma negativa las personas que estaban acostumbradas a una vida social activa, aunque terminan aceptándolo con resignación. Resignación es una de las palabras que mejor describe el estado de ánimo de las cuidadoras, tanto por la situación que les ha tocado vivir como por las consecuencias que implica en su vida. Algunas de las respuestas habituales ante la pregunta de cómo viven la situación son: "condenada", "sufrimiento", "obligada", "cansancio".

A pesar de las dificultades, se buscan válvulas de escape para poder desconectar, sin alterar significativamente la rutina diaria. Así, la mayoría se llevan a cabo dentro de la propia casa, o implican salidas muy puntuales y limitadas en el tiempo. Las actividades de larga duración son más difíciles de realizar, ya que implican largos períodos de tiempo en que la persona dependiente debe estar sola -lo que no siempre es posible- o se deben buscar alternativas que faciliten la substitución. Entre las actividades que se valoran más destacan los cursos de yoga o de idiomas, que permiten relacionarse con otras personas.

El momento del día propicio para disponer de una cierta intimidad es después de la cena, cuando la persona dependiente se ha acostado y las cuidadoras pueden aprovechar para hacer manualidades, descansar en el sofá, ver la televisión, o no hacer ninguna actividad concreta. Destaca la relevancia que han adquirido las nuevas tecnologías, especialmente el acceso a Internet, así como la introducción en las redes sociales.

A pesar de la importancia de estas actividades, la sensación generalizada es la imposibilidad de desconectar, de estar permanentemente pendiente de otra persona. Es interesante destacar el rol que juegan, en este sentido, las nuevas tecnologías ya que si bien por un lado, como se ha comentado anteriormente, favorecen el contacto y el vínculo permanente; por otra parte, dificultan las posibilidades de desconexión. Este es el caso de la aplicación WhatsApp, en el que los mensajes instantáneos y la posibilidad de enviar fotografías otorgan, en caso de estar separados, la sensación de tranquilidad, pero, contrariamente, dificultan la desconexión.

\section{Las cuidadoras no familiares}

En el caso de las cuidadoras no familiares, existe una serie de particularidades que justifica que se analicen específicamente: la precariedad de los trabajos de cuidado y el servicio doméstico; la prevalencia de las relaciones laborales informales; la vulnerabilidad de las personas extranjeras en situación administrativa irregular, etc. 
La mayoría de las entrevistadas se han dedicado exclusivamente a las tareas de cuidado desde su llegada al país de destino. Así, la mayoría hace unos 10 años que cuidan personas dependientes, a pesar de que su expectativa, antes de iniciar el movimiento migratorio, no era trabajar en este ámbito. Muchas expresan la frustración que les supuso descubrir las limitaciones que les impedirían trabajar de manera regular, debido a la legislación de extranjería, así como no poder acceder a ciertos sectores de empleo.

Creo que hay otras personas que van con un objetivo [encontrar otro trabajo] y al ver que este objetivo no está como uno pensaba la verdad que uno se viene abajo, se frustra. Pero yo soy de las que tal vez me frustré al principio pero todavía tengo esta esperanza (CNF5).

Las actividades de las cuidadoras suelen ser diversas y no difieren excesivamente de las familiares. En los casos que se cuida a personas dependientes con más limitaciones, las cuidadoras desarrollan todo tipo de tareas: domésticas, cotidianas y de cuidados y atención. En cambio, en el caso de las personas con mayor autonomía, las tareas más habituales son las domésticas y las cotidianas, mientras que las de cuidado personal se limitan a apoyos puntuales ya que las personas, si son autónomas, prefieren hacerlas por sí mismas.

Las cuidadoras manifiestan la elevada responsabilidad de su tarea, que no se corresponde con la remuneración que perciben. También destacan la creciente especialización de las tareas de cuidado, que requieren conocimientos y habilidades cada vez más complejas, que en algunos casos no se está capacitado para llevar a cabo. La precariedad asociada a estas actividades, especialmente con la notable incidencia del trabajo sumergido, dificulta la oferta de trabajadores autóctonos, y provoca que mayoritariamente sean ocupados por extranjeras en situación irregular.

Todas las entrevistadas ponen de manifiesto dificultades iniciales, tanto para relacionarse con la persona dependiente como con el resto de familiares, así como también para situarse en un nuevo entorno y en las actividades y funciones que deben desarrollar. Este no es un proceso sencillo para ninguno de los actores involucrados, y, lógicamente, aparecen conflictos de convivencia que se deben ir limando. "Y mira, ya después ella se había encariñado, primero no quería, ¿eh? ¡Buf! “¡Fea!, ¡qué se vaya, qué se vaya!", y ya después quería que yo la cuidara" (CNF1).

Las cuidadoras desarrollan estrategias para facilitar los procesos de adaptación mutua. Algunas destacan actitudes como la prudencia, la escucha y el respeto, así como no querer imponer cambios drásticos en el funcionamiento ordinario de los hogares, sino establecer de manera progresiva aquellos cambios que se consideren oportunos:

He tratado de adaptarme a ellos para que ellos no sientan como que yo les he llegado a imponer. Porque ellos ese era el miedo que tenían, ellos pensaban que iría a llegar alguien y los iba a hacer y deshacer en casa de ellos (CNF7).

En este sentido, un aspecto que se considera muy importante es contar con el apoyo de los miembros de la familia, ya que una relación cordial y atenta facilita mucho la superación de las dificultades propias del trabajo y las necesidades de las personas cuidadoras. La familia también puede apoyar en las actividades cotidianas, algunas de las cuales conllevan dificultades físicas importantes.

Sin embargo, algunos familiares consideran que, como están remunerando por estas actividades, esto les exonera de las responsabilidades de cuidado y les da derecho a tratar a las cuidadoras estrictamente con criterios propios de una relación contractual. La mercantilización de las tareas de cuidado implica una relación de poder entre quien contrata y quien es contratado $\mathrm{y}$, en el seno de esta relación desigual, algunas familias entienden que las cuidadoras deben estar permanentemente trabajando dentro del domicilio, llevando a cabo constantemente todo tipo de tareas. Por suplir la falta de apoyos de otros familiares, sólo en casos esporádicos, algunas personas han establecido relaciones de confianza con vecinos y vecinas, tejiendo redes de solidaridad mutuas.

Por otra parte, la mayoría de las entrevistadas considera que están bien preparadas para ejercer su trabajo, a pesar de que no han realizado cursos de formación. El principal motivo para no haberse formado son las dificultades que implica su actividad diaria, que no les permite estar mucho tiempo fuera del domicilio. Además, se expresa el desconocimiento de la oferta formativa. Sin embargo, algunas mues- 
tran interés por formarse, pero chocan con la negativa de las familias y/o de la persona a quien cuidan. Habitualmente esto implica conflictos con los familiares, por lo que, en otras ocasiones, las cuidadoras no se plantean la posibilidad de participar. Además, como se verá a continuación, las disputas con los familiares para participar en actividades fuera del hogar son habituales, por lo que se seleccionan minuciosamente el tipo de actividades extraordinarias -que implican la salida del domicilio- que negociarán con la familia.

Estuve asistiendo nada más un tiempo, porque con la abuela tuve problemas... entonces no podía asistir... Con ella, porque, vale, era de una hora y media y ui! Aquella señora, cuando yo regresaba, me decía de todo, entonces a mí me fastidiaba, porque imagínese, que era aquí, en el mismo pueblo, y yo tardaba una hora y media y ella estaba (CNF5).

Del mismo modo que las cuidadoras familiares, las cuidadoras no familiares perciben que su tarea no está bien valorada socialmente, a pesar de que es una cuestión que no se han planteado con excesivo detenimiento. Sin embargo, algunas expresan incredulidad acerca de la diferente consideración social que tienen en comparación, por ejemplo, con los maestros.

Mira, valorado creo que no, porque nosotros a veces subestimamos a las personas que se encargan de cuidar a nuestros padres, que son casi nuestra familia, porque son los que están pendientes de... más que nosotros, yo vivo pendiente de él, de que tiene que tomarse el medicamento, vivo pendiente de a qué hora tiene al médico (CNF2).

Por lo que respeta al reconocimiento y al trato que reciben de las personas que cuidan y de sus familiares, las cuidadoras perciben una cierta satisfacción de las personas que cuidan, a pesar de las dificultades iniciales, especialmente en aquellos casos en que las familias demandan una cuidadora por primera vez. Naturalmente, este es un momento complicado para las familias, por todo lo que supone la entrada en el domicilio de una persona desconocida. También son frecuentes los prejuicios y los estereotipos: las cuidadoras no familiares experimentan recelos por su condición de extranjeras y por un imaginario social acerca de las mujeres latinoamericanas.
Porque ella, más que todo, tiene una idea como del dinero, mucho, piensa que uno le va a venir a robar, entonces yo le explicaba que yo no le venía a robar, que nada más le venía a cuidar y a hacer cosas que ella ya no estaba para hacerlas. Que iba a estar en su casa y que yo no le iba a tocar absolutamente nada (CNF5).

Otra de las implicaciones derivadas de la falta de consideración social son determinados episodios en que las cuidadoras perciben un trato discriminatorio por parte de algunos familiares: un trato que se traduce de diferentes maneras, como la ignorancia, el desprecio, un trato irrespetuoso, entre otros.

Sólo le hablaban a él y yo... o sea, en ese momento desaparecía, y me sentía tan mal, [...] el chico éste llegaba [...], saludaba al señor, a uno ni le veía, no sé, a veces, lo relegan a uno, a un plano que, un plano de invisibilidad, o sea, tú no estás, sólo está él, y basta, y eso sí sabe mal (CNF2).

Sin embargo, también se cuentan casos más favorables de familias que valoran la labor y la importancia de las personas cuidadoras, y que las acogen sin prejuicios, haciéndolas partícipes de lo cotidiano, como si se tratara de su propio hogar.

En salario no estoy tan mal que se diga, pero ahí, todo, el trato, como son ellos, yo no me puedo quejar, porque son muy... excelentes. Porque ellos tratan de... son bien comprensivos, los hijos, igual, entonces ellos entienden, y yo siento que a comparación de otras que sí, les va bastante mal (CPROF7).

Por lo que respecta a las condiciones laborales, en general, la condición de extranjeras en situación irregular afecta de manera crucial. En cuanto al contrato, sólo dos personas disponen de contrato de trabajadoras del hogar y, obviamente, a pesar de tratarse de un sector precarizado, sus condiciones son mejores que las de quienes trabajan sin contrato. En cuanto al salario, teniendo en cuenta que se trata de personas que trabajan como internas las 24 horas del día, los salarios oscilan entre los 600 y los 900 euros mensuales. En algunos casos, el salario es el mismo, tanto si se cuida una persona como a dos, y no se modifica en caso de fallecimiento de alguno de los miembros. La mayoría consi- 
dera que están mal pagadas, ya que desarrollan tareas que pocas personas autóctonas quieren realizar y con unas condiciones laborales duras que les afectan en el ámbito personal. Sin embargo, pocas están dispuestas a reivindicar mejoras, ya que son conscientes de que su exigua posición de fuerza limita enormemente su capacidad de negociación. Algunas han intentado conseguir mejoras, pero el proceso les ha comportado muchas tensiones. Algunos familiares se muestran poco predispuestos a escuchar las peticiones de incrementos salariales, incluso en los casos en que hace más de cinco años que se dispone de una vinculación laboral estable y se percibe el mismo salario. Y, en otros casos, porque, si la persona dependiente se entera de las demandas de mejora salarial de la cuidadora, lo interpretan negativamente y reaccionan con hostilidad, acusándolas de "preocuparse sólo de su dinero".

El alojamiento en el domicilio de la persona dependiente es un elemento más de la precariedad en que se encuentran las cuidadoras, ya que el fallecimiento de la persona que cuidan implica, automáticamente, la pérdida del trabajo, pero también de la vivienda. Esto supone un interés por encontrar rápidamente trabajo (así como más predisposición a aceptar cualquier condición). Como afirma una cuidadora: "Me quedo sin trabajo, me quedo sin nada" (CNF4).

Finalmente, otra de las situaciones que se pueden dar ocasionalmente es el incumplimiento de algunos acuerdos en torno a futuros incrementos salariales, que se formulan habitualmente al inicio de la relación laboral y que se aplazan a la espera de ver cómo se desarrollan los acontecimientos. Cuando llega el momento en que se plantea esta posibilidad, a menudo las cuidadoras reciben negativas y se encuentran inmersas en disputas con los familiares, que rápidamente se abandonan ante la sensación de impotencia.

Existe una insatisfacción alrededor del salario, que provoca que muchas personas aspiren a regularizar su situación; un hecho que les permitiría, según ellas, disponer de más derechos y plantearse la entrada a otros ámbitos laborales con mejores condiciones. En pocos casos se dispone de vacaciones, y no suelen ir más allá de quince días al año. Además, salvo excepciones, las personas no reciben todo el sueldo que les correspondería, sino la mitad. En otros casos, no se percibe ninguna retribución.
Durante el transcurso del día, las cuidadoras suelen disponer de algunas horas libres. En algunos casos, por ejemplo, entre tres y cuatro horas diarias entre semana, pero la mayoría oscilan entre una y dos horas diarias. A lo largo de este tiempo, sin embargo, no se produce una separación absoluta con las personas a quienes se cuida, sino que se puede estar conectado mediante el teléfono móvil, lo que genera recelos entre algunas cuidadoras, que se niegan a utilizarlo.

En cuanto a los impactos en la vida personal, predominan las consecuencias en la salud emocional. Así, destaca la angustia que padecen las personas en situación administrativa irregular (temores a inspecciones policiales, dificultades para regularizarse, desconocimiento de las normativas, vulnerabilidad social, titularidad de menos derechos y garantías, etc.). Así mismo, la sensación de provisionalidad constante ante las trayectorias laborales fragmentadas e inestables, que obligan a encontrar nuevos trabajos periódicamente, y por las consecuencias que implica la pérdida de la vivienda.

Pero hay más elementos que acentúan aún más esta situación. La dureza de las tareas que se llevan a cabo, ya sea por el desgaste físico provocado por las actividades del hogar y las actividades cotidianas, como también por el hecho de tener que trabajar con personas que exigen una atención continuada e intensa, como son las personas con altos grados de dependencia. Además de la intensidad, algunas de estas actividades y tareas implican llevar a cabo, como ya se ha visto, trabajos incómodos y desagradables, como las relacionadas con la higiene personal, así como relaciones complicadas con personas que tienen bastante limitadas sus facultades físicas y mentales.

Por todo ello, las cuidadoras viven con ambivalencia su trabajo, des de una relativa satisfacción, hasta el descontento. En términos generales se detecta más insatisfacción, sobre todo por las condiciones laborales y las características del trabajo.

\section{Conclusiones}

Este estudio de caso pone de manifiesto algunas de las características del modelo mediterráneo de bienestar (Ferrera, 1996), en que las mujeres asumen un papel fundamental en la provisión del bienestar, cubriendo buena parte 
de las necesidades que en otros modelos asume el Estado bajo los principios de universalidad y justicia social. Sin embargo, este escenario parece difícil de mantener en los próximos años, por la aparición de nuevos riesgos sociales y el paso de la sociedad industrial a una postindustrial (Taylor-Gooby, 2004).

En el caso analizado, la situación de las cuidadoras familiares y las cuidadoras no familiares presenta una serie de regularidades compartidas, aunque también diferencias. Entre las conclusiones comunes, hay que destacar la dedicación absoluta de las mujeres a los cuidados. Una dedicación que, en algunos casos, implica haber trabajado consecutivamente en el cuidado de distintas personas, con lo cual, la sensación de sobrecarga es generalizada. Además de la elevada intensidad y dedicación, cabe añadir las consecuencias negativas para la salud (Río Lozano, 2014), que se manifiestan en problemas físicos, pero también psicológicos, que mellan de manera muy considerable la calidad de vida de las cuidadoras (Mateo Rodríguez et al., 2000; Larrañaga et al., 2008). Sin duda, la pregunta, quién cuida a las cuidadoras, es bien pertinente (Casado, 2016), ya que las ayudas que los familiares reciben para cuidar a las personas dependientes, ya sean transferencias monetarias o servicios complementarios al domicilio, son, en opinión de las entrevistadas, insuficientes.

En el caso de las cuidadoras no familiares, la irregularidad es un factor determinante que explica las malas condiciones sociales y laborales que sufren. El salario y las condiciones laborales son muy deficientes, con lo cual tampoco disponen de tiempo para desconectar de un trabajo tan absorbente como son los cuidados. En ambos casos, la situación de las cuidadoras repercute negativamente en su calidad de vida pero, al mismo tiempo, en la atención que ofrecen a las personas dependientes, con lo cual serían necesarias medidas que ampliaran la oferta de prestaciones para atender a las personas dependientes. De esta forma se mejoría su calidad de vida, y se evitarían situaciones personales especialmente duras como las que afrontan las cuidadoras.

\section{Referencias bibliográficas}

Abellán García, A., Esparza Catalá, C. y Pérez Díaz J. (2011). Evolución y estructura de la población en situación de dependencia. Cuadernos de Relaciones Laborales, 29(1), 43-67.

Aguilar Hendrickson, M.A. (2014). Hacia un replanteamiento de los servicios sociales en España. Documentación social, 175, 35-64.

Anderson, B. (2014). Nations, migration and domestic labor: The case of the UK. Women's Studies International Forum, 46(5).

Bettio, F., Simonazzi, A. y Villa, P. (2006). Change in care regimes and female migration: the 'care drain' in the Mediterranean. Journal of European Social Policy, 16, 271-285.

Campo Ladero, M.J. (2000). Apoyo informal a las personas mayores y el papel de la mujer cuidadora. Madrid: Centro de Investigaciones Sociológicas.

Carrasquer, P. (2013). El redescubrimiento del trabajo de cuidados: algunas reflexiones desde la sociología. Cuadernos De Relaciones Laborales, 31(1), 91-113. doi:10.5209/rev_CRLA.2013.v31.n1.41633

Carretero, S., Garcés, J. y Rodenas, F. (2006). La sobrecarga de las cuidadoras de personas dependientes: Análisis y propuesta de intervención psicosocial. Valencia: Tirant lo Blanch.

Casado, M.I. (2016). ¿Quién cuida a las cuidadoras? Boletín ECOS, 37, Madrid: Fuhem.

Colectivo IOÉ. (2005). El cuidado de personas mayores dependientes realizado por ciudadanos inmigrantes en la Comunidad de Madrid. En Cuidado a la dependencia e inmigración. Madrid: IMSERSO

Esping-Andersen, G. (1993). Los tres mundos del Estado del Bienestar. València: Ed. Alfons el Magnànim.

Ferrera, M. (1996). The southern model of welfare state in social Europe. Journal of European Social Policy, 6(1), 17-37.

Fuentes, M.C. y Moro, L. (2014). Trabajo Social y cuidadores informales: análisis de la situación actual y propuesta de intervención. Trabajo Social Hoy, 71, 43-62. doi:10.12960/TSH.2014.0002.

Generalitat de Catalunya. (2008). Bases per al suport als cuidadors i les cuidadores no professionals. Col·lecció: Papers d'acció social, 1. Departament d'Acció Social i Ciutadania.

IMSERSO. (2005). Cuidados a las Personas Mayores en los Hogares Españoles. El entorno familiar. Madrid: Imserso, 
Lagarde, M. (2004). Mujeres cuidadoras: entre la obligación y la satisfacción. Congreso Internacional SARE 2003. Cuidar cuesta: costes y beneficios del cuidado, Emakunde-Instituto Vasco de la Mujer y Comunidad Europea, Vitoria-Gasteiz.

Larrañaga, I., Martín, U., Bacigalupe, A., Begiristáina, J.M., Valderrama, Ma J. y Arregi, B. (2008). Impacto del cuidado informal en la salud y la calidad de vida de las personas cuidadoras: análisis de las desigualdades de género. Gaceta Sanitaria, 22(5), 443-450.

Mateo Rodríguez, I., Millán Carrasco, A., García Calvente, M., Gutiérrez Cuadra, P., Gonzalo Jiménez, E. y López Fernández, L. (2000). Cuidadores familiares de personas con enfermedad neurodegenerativa: Perfil, aportaciones e impacto de cuidar. Atención Primaria, 26(3), 139-144.

Navarro, V. (2002). Bienestar insuficiente, democracia incompleta. Barcelona: Anagrama.

Río Lozano, M. (2014). Desigualdades de género en el cuidado informal y su impacto en la salud. (Tesis inédita, Universidad de Granada).

Rodríguez Cabrero, G. (2011). Políticas sociales de atención a la dependencia en los Regímenes de Bienestar de la Unión Europea. Cuadernos de Relaciones Laborales, 29(1), 13-42.

Rogero, J. (2009a). Los tiempos del cuidado. El impacto de la dependencia de los mayores

en la vida cotidiana de sus cuidadores. Madrid: IMSERSO.

Rogero, J. (2009b). Distribución en España del cuidado formal e informal a las persones de 65 y más años en situación de dependencia. Revista Española de Salud Pública, 83(3), 393-405

Taylor-Gooby, P. (2004). New risks, new welfare. Oxford: Oxford University Press.

Tobío, C., Agulló Tomás, Mª S., Gómez, Mª V. y Martín Palomo, M $M^{\mathrm{a}}$ T. (2010). El cuidado de las personas. Un reto para el siglo XXI. Barcelona: Obra Social, Fundación "la Caixa", 28.

Torns, T., Borrás, V., Moreno, S. y Recio, C. (2012). El trabajo de cuidados. Un camino para repensar el bienestar. Papeles de relaciones ecosociales y cambio global, 119, 93-101.

Torns, T. (2008). El trabajo y el cuidado: cuestiones teórico-metodológicas desde la perspectiva de género. EMPIRIA: Revista de Metodología de Ciencias sociales, 15, 53-73.

Vilà, A. (2011). Serveis socials: aspectes històrics, institucionals i legislatius. Barcelona: Editorial UOC. 